\title{
OPEN A novel AlGaN/GaN heterostructure field-effect transistor based on open-gate technology
}

\author{
Yang Liu ${ }^{1}$, Yuanjie Lv ${ }^{2}$, Shuoshuo Guo ${ }^{1}$, Zhengfang Luan ${ }^{3}$, Aijie Cheng ${ }^{3}$, Zhaojun Lin ${ }^{1 凶}$, \\ Yongxiong Yang ${ }^{1}$, Guangyuan Jiang ${ }^{1}$ \& Yan Zhou ${ }^{1}$ \\ In this study, a novel AIGaN/GaN heterostructure field-effect transistor based on open-gate \\ technology was fabricated. Sample transistors of different structures and sizes were constructed. \\ Through measurements, it was found that by changing the width of the opening, the threshold \\ voltage of the device could be easily modulated across a larger range. The open-gate device had two \\ working modes with different transconductance. When the gate-source voltage $V_{\mathrm{GS}} \leq-4.5 \mathrm{~V}$, only \\ the open region was conductive, and a new working mechanism modulated the channel current. \\ Corresponding theoretical analysis and calculations showed that its saturation mechanism was \\ related to a virtual gate formed by electron injection onto the surface. Also, the gate-source voltage \\ modulated the open channel current by changing the channel electron mobility through polarization \\ Coulomb field scattering. When used as class-A voltage amplifiers, open-gate devices can achieve \\ effective voltage amplification with very low power consumption.
}

The AlGaN/GaN heterostructure field-effect transistor (HFET), a type of wide-bandgap semiconductor electronic device, has the advantages of high breakdown voltage and high electron mobility, which has led to it being widely used in high frequency and high power applications ${ }^{1-6}$. Whether for switching devices or RF power devices, the threshold voltage $\left(V_{\text {th }}\right)$ is a very important parameter. However, the common methods to change the $V_{\text {th }}$ of $\mathrm{AlGaN} / \mathrm{GaN}$ HFETs are relatively complex. It is usually necessary to change the epitaxial structure of the material or introduce new process steps, such as changing the thickness, doping concentration or Al composition of the barrier layer, plasma treatment of the material surface, growing an insulating layer with a certain thickness above the barrier layer, etc ${ }^{7-12}$. It is extremely difficult to change the $V_{\mathrm{th}}$ of the device across a large range by simply adjusting the device structure.

Additionally, polarization Coulomb field (PCF) scattering is an important scattering mechanism in AlGaN/ GaN HFETs. It has an important influence on the gate region electron mobility, gate-source resistance, extrinsic transconductance, linearity and other characteristics of the devices ${ }^{13-17}$. To a certain extent, $V_{\text {th }}$ can reflect the voltage range in which the gate-source voltage $\left(V_{\mathrm{GS}}\right)$ can effectively modulate the drain-source current $\left(I_{\mathrm{DS}}\right)$, and PCF scattering is related to both $V_{\mathrm{GS}}$ and $I_{\mathrm{DS}}$. Therefore, it should be entirely possible to design a new device structure using the mechanism of PCF scattering to achieve large-scale modulation of $V_{\text {th }}$ in AlGaN/GaN HFETs.

In this paper, a novel AlGaN/GaN HFET which had an open gate structure and a new working mechanism was designed. The open-gate device had two working modes with different modulation abilities. Under the condition that the gate region two-dimensional electron gas (2DEG) was depleted, the open region electron mobility could be modulated by PCF scattering, to enable the modulation of $I_{\mathrm{DS}}$ by $V_{\mathrm{Gs}}$. After the introduction of the open gate structure, a simple method of changing the width of the gate opening could be used to modulate $V_{\text {th }}$ across a larger range. As class-A voltage amplifiers, open-gate devices can achieve effective voltage amplification with very low power consumption. The open-gate device in this paper had two independent gates, which would increase the complexity of device packaging, but also provided an extra degree of freedom for its application in the circuit. In addition, a new device structure can be designed to connect two gates to the same pad to avoid complex packaging.

\footnotetext{
${ }^{1}$ School of Microelectronics, Institute of Novel Semiconductors, Shandong University, Jinan 250101, China. ${ }^{2}$ National Key Laboratory of Application Specific Integrated Circuit (ASIC), Hebei Semiconductor Research Institute, Shijiazhuang 050051, China. ${ }^{3}$ School of Mathematics, Shandong University, Jinan 250100, China. ${ }^{\circledR}$ email: linzj@sdu.edu.cn
} 

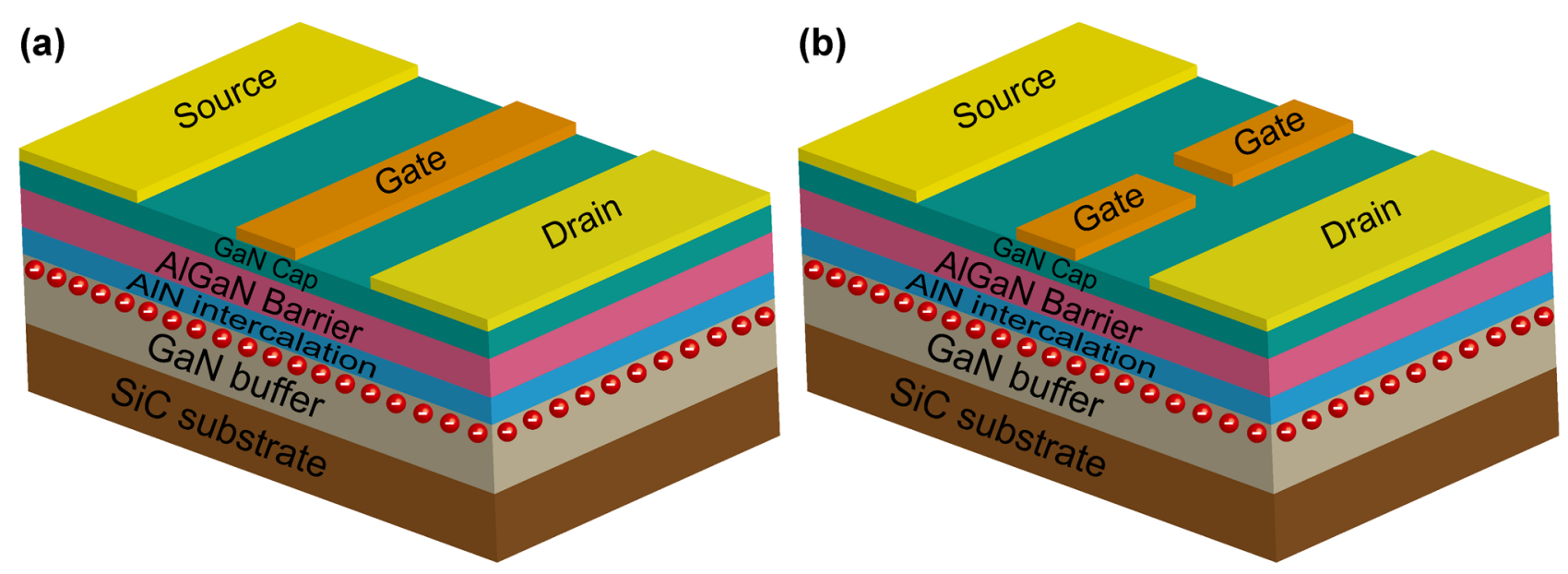

Figure 1. Schematic diagram of the structure of (a) normal device and (b) open-gate device. The figure was generated by Adobe Illustrate CC 2019 (https://www.adobe.com/cn/products/illustrator.html).

\section{Results and discussion}

Analysis of device characteristics. Four AlGaN/GaN HFETs with different structures and sizes were prepared, and the structural difference between normal device and open-gate device is shown in Fig. 1. For these four samples, the gate length $\left(L_{\mathrm{G}}\right)$ was $40 \mu \mathrm{m}$, the total channel width $(W)$ was $100 \mu \mathrm{m}$, and the gate-source distance $\left(L_{\mathrm{GS}}\right)$ and gate-drain distance $\left(L_{\mathrm{GD}}\right)$ were both $6 \mu \mathrm{m}$. Sample 1 was a normal device, with an unopened gate. Samples 2,3 , and 4 were all devices with an opening in the middle of the gate, and the opening widths $\left(W_{\mathrm{O}}\right)$ were $3 \mu \mathrm{m}, 4 \mu \mathrm{m}$, and $5 \mu \mathrm{m}$, respectively. The large device size is related to the working mechanism of the open-gate device, which will be discussed in detail later.

The output characteristics with logarithmic y-axis and standard y-axis of the four samples are shown in Fig. 2. It can be clearly seen that for these four samples, $V_{\mathrm{GS}}$ had a good ability to modulate $I_{\mathrm{DS}}$. Even for open-gate samples, $I_{\mathrm{DS}}$ could still be modulated by $V_{\mathrm{GS}}$ until the current was turned off. When $V_{\mathrm{GS}} \leq-4.5 \mathrm{~V}$, the gate bias of open-gate devices could still effectively modulate the current across a wide range, which is not observed in the normal device. With an increase in $W_{\mathrm{O}}$, the sample $I_{\mathrm{DS}}$ could be modulated over a larger $V_{\mathrm{GS}}$ range. As shown in Fig. 2e, when $V_{\mathrm{GS}}=-4 \mathrm{~V}$, there is a kink near the linear-saturation crossover of the output characteristic curve. The specific mechanism of kink effect is still controversial, but it is generally believed that this kink is related to electron traps in AlGaN/GaN heterojunction materials ${ }^{18-20}$. With the increase of $V_{\mathrm{DS}}$, the channel electrons are first captured by the trap and then escape from the trap, resulting in the channel current first decreasing and then increasing. It should be noted that for the material in this paper, due to the low electron trap density, the kink effect can be obvious only when the channel 2DEG density $\left(n_{2 \mathrm{D}}\right)$ is low. For open-gate devices, since the open region always maintains a high $n_{2 \mathrm{D}}$, which will be described in detail later, there is no obvious kink effect.

As an important parameter, $V_{\text {th }}$ can reflect the voltage range in which $V_{\mathrm{GS}}$ can effectively modulate $I_{\mathrm{DS}}$. Using the constant current method, when the saturation current reaches the order of $10^{-8} \mathrm{~A}$, the corresponding gate bias is defined as $V_{\mathrm{th}}$. The values obtained were $V_{\mathrm{th}}=-4.5,-6,-7$, and $-8 \mathrm{~V}$, for the four samples labeled 1,2 , 3 , and 4, respectively. This is consistent with what is shown in Fig. 2. It was found that open-gate devices had a lower $V_{\text {th }}$ than normal devices. As $W_{\mathrm{O}}$ increased, $V_{\text {th }}$ changed in the negative direction, so the $I_{\mathrm{DS}}$ of the samples could be modulated over a larger gate voltage range. In other words, the $V_{\text {th }}$ of the devices could be modulated simply by changing $W_{\mathrm{O}}$.

The capacitance-voltage $(\mathrm{C}-\mathrm{V})$ characteristics with standard $y$-axis and logarithmic $y$-axis of four samples are shown in Fig. 3. It can be seen that the $\mathrm{C}-\mathrm{V}$ curves of the four samples are almost the same when $V_{\mathrm{GS}}>-4.5 \mathrm{~V}$. This is because the capacitance of the device obtained from the $\mathrm{C}-\mathrm{V}$ measurement in this voltage range only corresponds to the region underneath the gate, and the gate areas of the four samples are very close. When the $V_{\mathrm{GS}}$ is about $-4.5 \mathrm{~V}$, the capacitance of the four samples decreases rapidly to a minimum, which means that the $2 \mathrm{DEG}$ underneath the gate region is almost depleted around $V_{\mathrm{GS}}=-4.5 \mathrm{~V}$. For the $2 \mathrm{DEG}$ in the open region, specific analysis will be carried out as follows.

With the change of gate bias, a lateral electric field may modulate 2DEG in the open region, which is observed in in-plane-gate field effect transistors (IPGFETs) ${ }^{21,22}$. However, for IPGFETs, the barrier layer below the gate is metallized by annealing, so that the gate and channel 2DEG are in the same plane, which further leads to a strong role of the lateral electric field. For AlGaN/GaN open-gate devices, since the gate and channel 2DEG are separated by an AlGaN barrier layer, the lateral electric field cannot be established directly as IPGFETs. However, the gate can still modulate channel 2DEG through the fringe electric field, which is similar to the AlGaAs/GaAs split-gate transistor ${ }^{23-26}$. According to the relevant research results of AlGaAs/GaAs split gate structure ${ }^{23-25}$, before the 2DEG underneath the gate is depleted, the gate bias will not affect the 2DEG in the open region. After that, due to the effect of the fringe electric field, the gate bias will change the amount of charge in the open region, which will be reflected in the $\mathrm{C}-\mathrm{V}$ characteristics. As shown by the $\mathrm{C}-\mathrm{V}$ curves with logarithmic $\mathrm{y}$-axis in Fig. 3, it can be seen that when $V_{\mathrm{GS}} \leq-4.5 \mathrm{~V}$, although the value is very small, the capacitance of the opengate device is significantly greater than that of the normal device, and the difference between them reflects the 

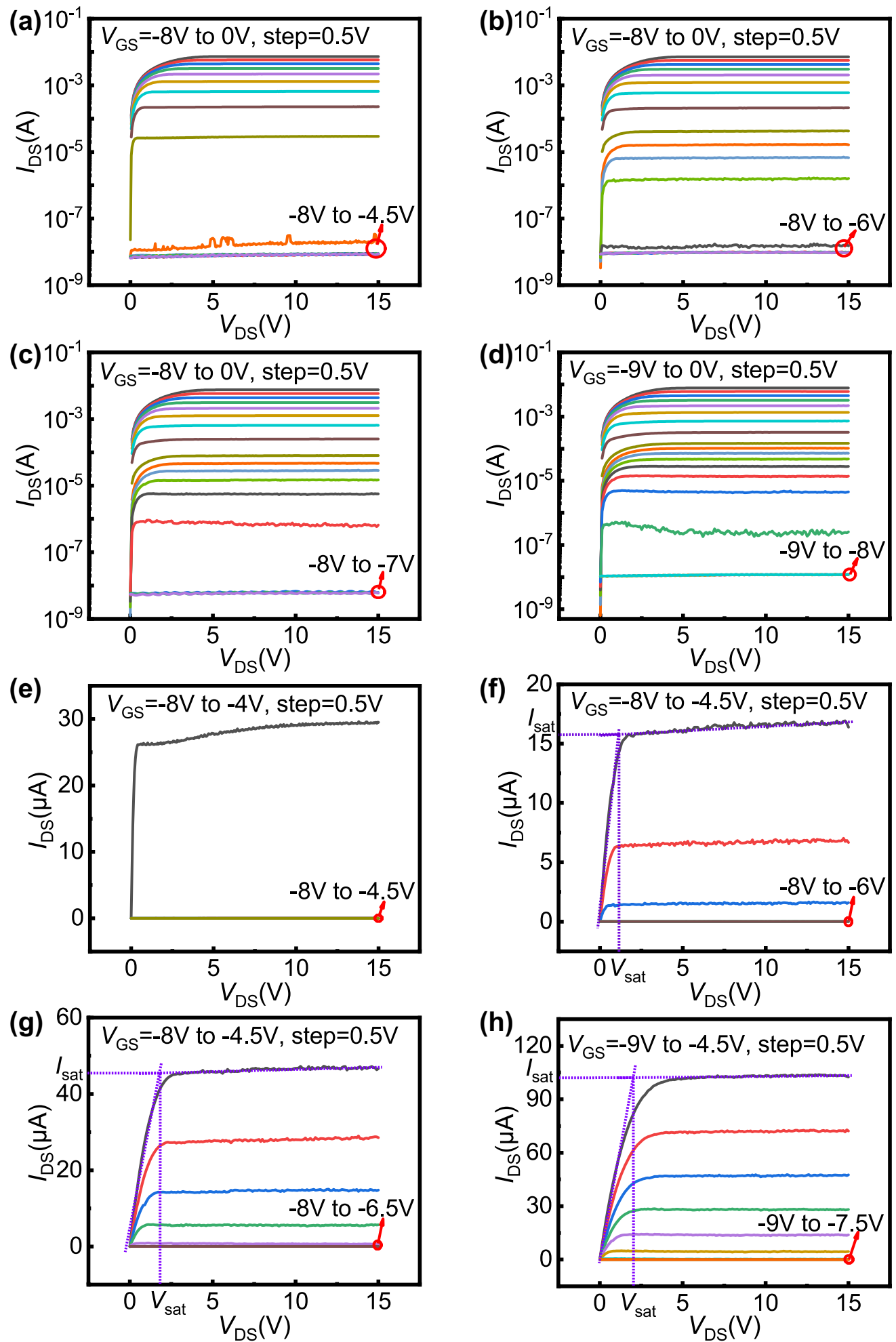

Figure 2. The measured output characteristics with logarithmic y-axis: (a) sample 1, (b) sample 2, (c) sample 3 , and (d) sample 4 . The measured output characteristics with standard y-axis at a specific range of gate bias: (e) sample 1, (f) sample 2, (g) sample 3, and (h) sample 4. 

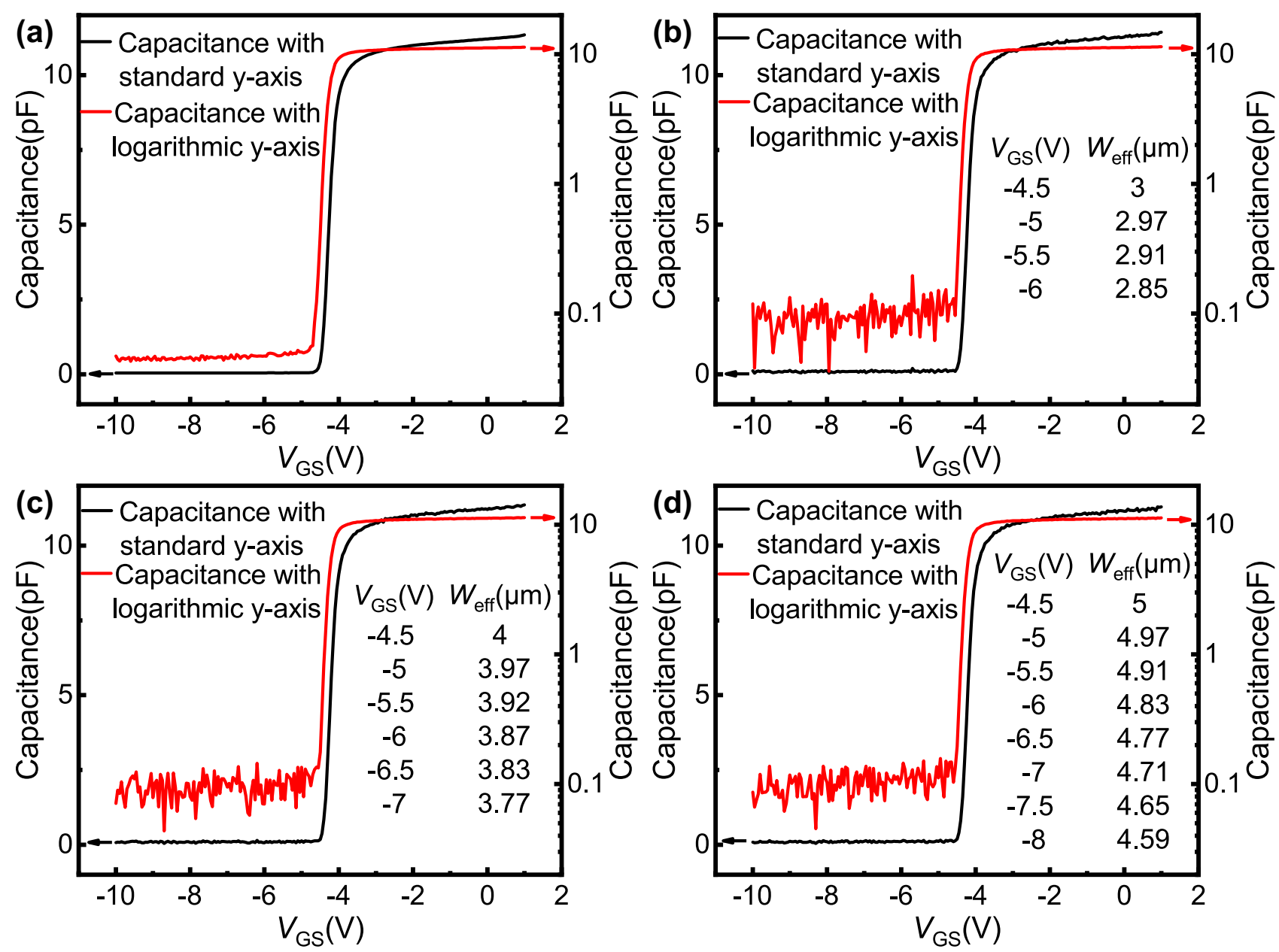

Figure 3. The measured $\mathrm{C}-\mathrm{V}$ characteristics with standard y-axis and logarithmic y-axis: (a) sample 1, (b) sample 2, (c) sample 3, and (d) sample 4. When measuring $\mathrm{C}-\mathrm{V}$ characteristics, different potentials are applied to gate and source respectively, and source and drain are not shorted. For open-gate devices, the capacitances of the two gates are measured separately and summed to obtain the capacitance shown in the figure. The effective widths of the open region under different gate bias calculated according to the $\mathrm{C}-\mathrm{V}$ characteristics are also marked in the figure.

$$
\Delta Q=\int_{V_{G S}}^{-4.5} C_{O} d V-\int_{V_{G S}}^{-4.5} C_{N} d V .
$$

Here, $C_{O}$ and $C_{N}$ are the capacitance of the open-gate device and normal device, respectively.

The studies on AlGaAs/GaAs split-gate devices show that the effective width of the open region $\left(W_{\text {eff }}\right)$ will narrow due to the consumption of 2DEG electrons by the fringe electric field ${ }^{23-25}$. As for whether the $n_{2 \mathrm{D}}$ of the narrowed open region changes with $V_{\mathrm{GS}}$, different studies show different results ${ }^{23,25,26}$. For the convenience of calculation, we adopt the results of Thornton et al. ${ }^{23}$ and assume that the $n_{2 \mathrm{D}}$ will remain constant as the $W_{\text {eff }}$ is reduced. When the fringe electric field consumes few 2DEG electrons in the open region, this assumption is obviously reasonable, and the narrowed $W_{\text {eff }}$ can be expressed as

$$
W_{e f f}=W_{O}-\Delta Q / e n_{2 D} L_{G}
$$

where $e$ is the absolute value of the electronic charge.

For open-gate devices, the specific value of $W_{\text {eff }}$ under different gate bias can be obtained and marked in Fig. 3. It can be seen that the change of $W_{\text {eff }}$ caused by fringe electric field is very small, which is not enough to pinch off the channel. Therefore, in addition to the modulation of fringe electric field on 2DEG in the open region, there must be a new working mechanism to modulate the current in the open region until it is turned off.

Based on the above analysis, it can be concluded that the open-gate samples have different working modes before and after $V_{\mathrm{GS}}=-4.5 \mathrm{~V}$. When $V_{\mathrm{GS}}>-4.5 \mathrm{~V}$, the whole channel, including the gate region and the open region, is conductive, and the open-gate device operates in mode 1 . At this time, as $V_{\mathrm{GS}}$ changes negatively, the $n_{2 \mathrm{D}}$ underneath the gate decreases, further leading to the decrease of $I_{\mathrm{DS}}$, which is the same as the working mechanism of normal devices. When the open-gate device operates in mode $2\left(V_{\mathrm{GS}} \leq-4.5 \mathrm{~V}\right)$, the 2DEG underneath the gate region becomes depleted, and only the open region is conductive. In this mode, as $V_{\mathrm{GS}}$ changes negatively, 

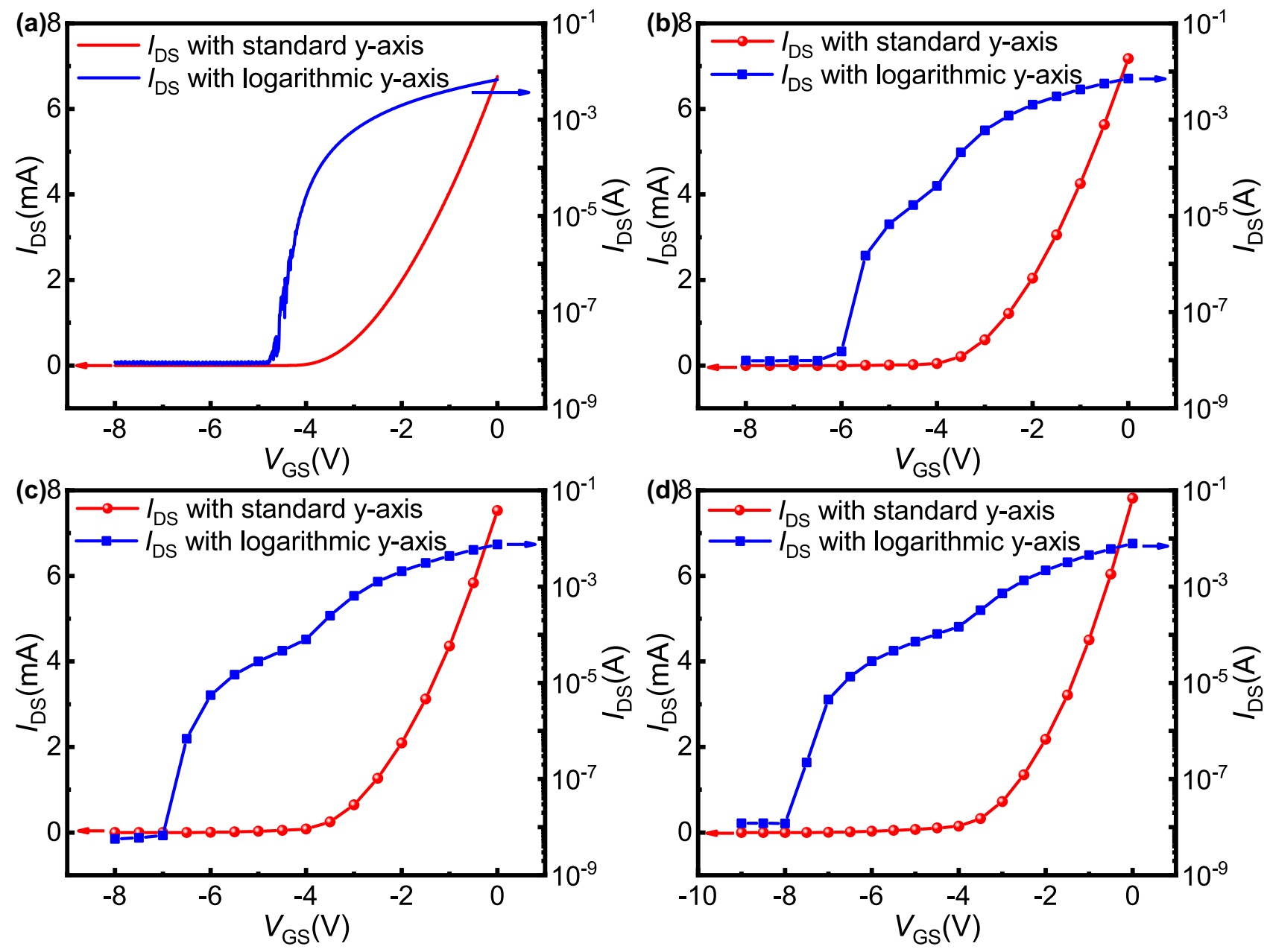

Figure 4. The measured transfer characteristics with standard y-axis and logarithmic y-axis: (a) sample 1, (b) sample 2, (c) sample 3, and (d) sample 4. The drain-source voltage is constant at $10 \mathrm{~V}$ during measurement. The transfer characteristics of open-gate devices are extracted from their output characteristics.

while the gate fringe electric field reduces $W_{\text {eff }}$ there is another mechanism affecting the channel electrons, which jointly modulate the channel current. The larger the width of the opening, the greater the initial current of mode 2 , and the more difficult it is to turn off. Therefore, $V_{\text {th }}$ changes negatively with an increase in $W_{\mathrm{O}}$.

To compare the modulation ability of the devices in the two working modes, it is necessary to calculate the transconductance before and after $V_{\mathrm{GS}}=-4.5 \mathrm{~V}$. Figure 4 shows the transfer characteristics with standard y-axis and logarithmic $y$-axis of four samples, where the drain-source voltage $\left(V_{D S}\right)$ is $10 \mathrm{~V}$. Since the two gates of the open-gate device are not connected, during the measurement of transfer characteristics, when a varying bias is applied to one gate, the same bias needs to be applied to the other gate at the same time, which cannot be achieved due to the instrument limitations. Therefore, we can only find the $I_{\mathrm{DS}}$ corresponding to different $V_{\mathrm{GS}}$ from the output characteristics under the condition of $V_{\mathrm{DS}}=10 \mathrm{~V}$, and extract them to form the transfer characteristics of open-gate devices. It can be clearly seen from Fig. 4 that the turn-off voltages of the four samples are basically consistent with the threshold voltages extracted by constant current method. The turn-off currents of the four samples are all in the order of $10^{-8} \mathrm{~A}$, which indicates that the current can be turned off completely even for the open-gate devices. The transconductance of the devices can be obtained by deriving the transfer characteristic curve. The calculated maximum transconductance $\left(g_{\mathrm{m} \text { max }}\right)$ of the open-gate samples before and after $V_{\mathrm{GS}}=-4.5 \mathrm{~V}$ are shown in Table 1 . It can be seen from the table that for open-gate devices, $g_{\mathrm{m}, \max }$ when $V_{\mathrm{GS}}>-4.5 \mathrm{~V}$ is much larger than that when $V_{\mathrm{GS}} \leq-4.5 \mathrm{~V}$, which indicates that the modulation ability of devices is significantly weakened when $V_{\mathrm{GS}} \leq-4.5 \mathrm{~V}$.

Figure 5 shows the variation of the measured gate leakage current $\left(I_{\mathrm{G}}\right)$ with $V_{\mathrm{GS}}$ for the four samples. It can be seen that the leakage current of these four samples has little difference. The maximum leakage current value of each sample is in the order of $10^{-8} \mathrm{~A}$, which is in the same order as the turn-off current and far less than the turn-on current. When $V_{\mathrm{GS}} \leq-4.5 \mathrm{~V}$, the leakage current of each sample remains almost unchanged. This shows that the effect of $I_{\mathrm{G}}$ on $I_{\mathrm{DS}}$ can be ignored when the device is on, and the modulation of gate bias on the current in the open region is not directly caused by $I_{\mathrm{G}}$. In addition, relevant research shows that the electrons leaking from the gate will be trapped in the empty surface state, thus forming a virtual gate ${ }^{27}$, which may affect the modulation of channel $n_{2 \mathrm{D}}$. However, the virtual gate formed by the above process should be located in the region between the 


\begin{tabular}{|l|l|l|l|}
\hline & \multicolumn{3}{|l|}{$g_{\mathrm{m}, \max }(\mathbf{S})$} \\
\cline { 2 - 4 } & Sample 2 & Sample 3 & Sample 4 \\
\hline$V_{\mathrm{GS}}>-4.5 \mathrm{~V}$ & $3.09 \times 10^{-3}$ & $3.38 \times 10^{-3}$ & $3.56 \times 10^{-3}$ \\
\hline$V_{\mathrm{GS}} \leq-4.5 \mathrm{~V}$ & $3.54 \times 10^{-5}$ & $5.17 \times 10^{-5}$ & $7.42 \times 10^{-5}$ \\
\hline
\end{tabular}

Table 1. The calculated maximum transconductance of samples 2, 3, and 4 .

gate and the drain ${ }^{27}$, which is distributed on both sides of the open region rather than directly above it. Therefore, even if the virtual gate caused by gate leakage exists, the $n_{2 \mathrm{D}}$ in the open region cannot be directly modulated.

Basic working mechanism. We hypothesize that for open-gate devices, the $V_{\mathrm{GS}}$ modulates the channel current in the open region by changing the electron mobility through the PCF scattering mechanism. In AlGaN/ GaN HFETs, there are multiple scattering mechanisms, such as dislocation (DIS) scattering, acoustic phonon (AP) scattering, polar optical phonon (POP) scattering, interface roughness (IFR) scattering, in addition to PCF scattering ${ }^{28-30}$. For a specific region in the channel, the scattering mechanisms other than PCF scattering are only correlated with $V_{\mathrm{GS}}$ through the $n_{2 \mathrm{D}}$ there. Since the $n_{2 \mathrm{D}}$ in the narrowed open region remains unchanged, it can be considered that the other scattering mechanisms in this region do not change with $V_{\mathrm{Gs}}$. The PCF scattering is not only dependent on the $n_{2 \mathrm{D}}$ in the open region, but also affected by the additional polarization charge density $(\sigma)$ underneath the gate region of the open-gate device. The polarization charges at the interface of the AlGaN/ $\mathrm{GaN}$ heterostructure are uniformly distributed before the device is fabricated ${ }^{31,32}$. However, after the device is fabricated, when $V_{\mathrm{GS}}$ is applied, the strain of the barrier layer underneath the gate changes owing to the existence of the inverse piezoelectric effect ${ }^{33}$. This non-uniform strain will lead to the uneven distribution of polarization charges, thereby generating additional polarization charges and scattering the channel electrons. This is what is known as PCF scattering ${ }^{34,35}$. For open-gate devices, as $V_{\mathrm{GS}}$ changes negatively, the additional polarization charge underneath the gate will increase, and the PCF scattering to the 2DEG electrons in the open region will increase accordingly. This will lead to a decrease in electron mobility and an increase in resistance in the open region. As a result, the current in the open region decreases as $V_{\mathrm{GS}}$ changes negatively, so that the gate bias modulates the channel current.

PCF scattering is essentially a Coulomb scattering, which reflects the interaction between additional polarization charges and channel electrons. In the $\mathrm{AlGaN} / \mathrm{GaN}$ heterostructure, the distribution of channel 2DEG is quantized and can be expressed by a wave function, written as $\Psi(x, y, z)=A^{-1 / 2} \psi(z) \exp \left(i k_{x} x+i k_{y} y\right)$ 36,37. Here, $A$ is the 2-D normalization constant, $k_{x}$ and $k_{y}$ are the components of the wave vector $\boldsymbol{k}$ in the $x$ and $y$ directions, respectively, $\psi(z)=\left(b^{3} z^{2} / 2\right)^{1 / 2} \exp (-b z / 2)$ is the Fang-Howard variational wave function, $b=\left(33 m^{*} e^{2} n_{2 D} / 8 \varepsilon_{0} \varepsilon_{s} \hbar^{2}\right)^{1 / 3}$ is the variational parameter, $m^{*}$ is the electron effective mass of the GaN material, $\hbar$ is the reduced Planck constant, $\varepsilon_{0}$ is the dielectric permittivity, and $\varepsilon_{\mathrm{s}}$ is the static dielectric constant of GaN.

For samples 2, 3, and 4, taking the open region as a benchmark, meaning that the additional polarization charge in the open region is considered to be zero, only the region underneath the gate has a negative additional polarization charge under negative $V_{\mathrm{Gs}}$. The two gates on both sides of the opening are symmetrical, and the voltages applied to the two gates are the same. Therefore, the additional polarization charges underneath the two gates are equal, expressed as $\sigma_{\mathrm{G} 1}=\sigma_{\mathrm{G} 2}=\sigma_{\mathrm{G}}$. As mentioned above, when $V_{\mathrm{GS}} \leq-4.5 \mathrm{~V}$, only the open region of the device is conductive. At this time, it is only necessary to consider the scattering effect of the additional polarization charges underneath the gate region on the electrons in the open region. This effect can be expressed by the PCF scattering potential, which can be written as ${ }^{35}$

$$
\begin{aligned}
V(x, y, z)= & -\frac{e}{4 \pi \varepsilon_{s} \varepsilon_{0}} \int_{-\frac{L_{G}}{2}}^{\frac{L_{G}}{2}} d x^{\prime} \int_{0}^{\frac{W-W_{e f f}}{2}} \frac{\sigma_{G}}{\sqrt{\left(x-x^{\prime}\right)^{2}+\left(y-y^{\prime}\right)^{2}+z^{2}}} d y^{\prime} \\
& -\frac{e}{4 \pi \varepsilon_{s} \varepsilon_{0}} \int_{-\frac{L_{G}}{2}}^{\frac{L_{G}}{2}} d x^{\prime} \int_{\frac{W+W_{e f f}}{2}}^{W} \frac{\sigma_{G}}{\sqrt{\left(x-x^{\prime}\right)^{2}+\left(y-y^{\prime}\right)^{2}+z^{2}}} d y^{\prime}
\end{aligned}
$$

The PCF scattering potential interacts with the electrons in the open region and scatters the electrons from the initial state $\boldsymbol{k}$ to the final state $\boldsymbol{k}^{\prime}$, which can be represented by the matrix element as ${ }^{35,36}$

$$
\begin{aligned}
M_{k \rightarrow k^{\prime}}= & A^{-1} \int_{0}^{\infty} \psi_{k^{\prime}}^{*}(z) \\
& \times\left[\int_{-\frac{L_{G}}{2}-L_{G S}}^{\frac{L_{G}}{2}+L_{G D}} d x \int_{\frac{W-W_{e f f}}{2}}^{\frac{W+W_{e f f}}{2}} V(x, y, z) \exp \left(-i q_{x} x-i q_{y} y\right) d y\right] \psi_{k}(z) d z \\
= & A^{-1} \int_{0}^{\infty} \psi_{k^{\prime}}^{*}(z)\left[V\left(q_{x}, q_{y}, z\right)\right] \psi_{k}(z) d z
\end{aligned}
$$



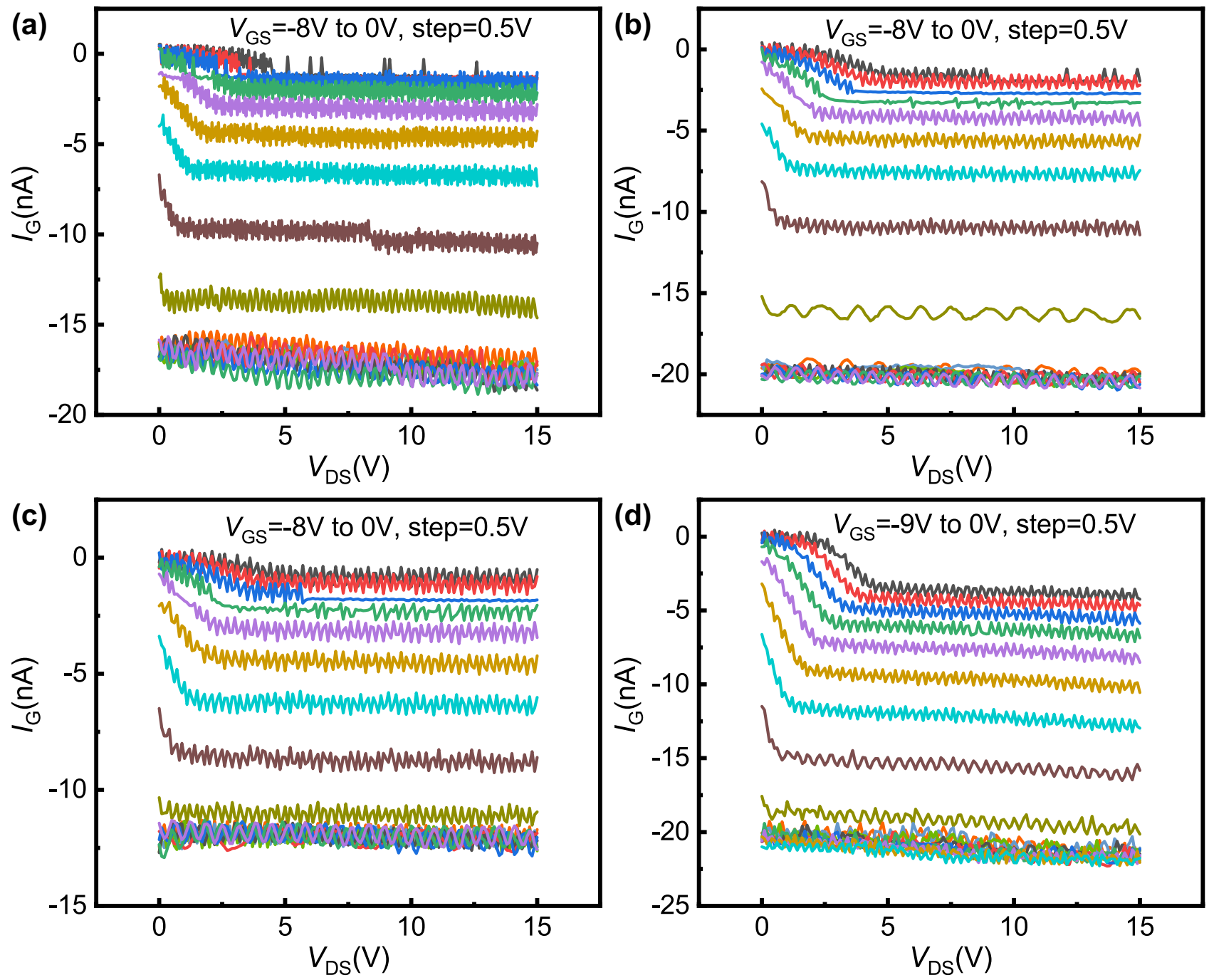

Figure 5. The measured gate leakage current as a function of the gate bias: (a) sample 1, (b) sample 2, (c) sample 3 , and (d) sample 4 .

Here, $q_{x}$ and $q_{y}$ are the components of the wave vector change $\boldsymbol{q}=\boldsymbol{k}^{\prime}-\boldsymbol{k}$ in the $x$ and $y$ directions, respectively. The wave vector change $\boldsymbol{q}$ satisfies $q=\left|2\left(2 m^{*} \hbar^{-2} E\right)^{1 / 2} \sin (\theta / 2)\right|$, where $E$ is the 2DEG electron energy, and $\theta$ is the scattering angle of the $2 \mathrm{DEG}$ electron from $\boldsymbol{k}$ to $\boldsymbol{k}^{\mathbf{2} 5}$.

After determining the matrix element, the derivation related to PCF scattering can be obtained by the standardized process (Supplementary Sect. 1).

Using the iterative calculation of PCF scattering ${ }^{38}$, the specific values of $\sigma_{\mathrm{G}}$ and various electron mobilities can be obtained for different $V_{\mathrm{GS}}$ values, and the calculated results are shown in Fig. 6 . It can be seen that as $V_{\mathrm{GS}}$ changes negatively, the absolute value of $\sigma_{\mathrm{G}}$ increases, which is consistent with the theory of the inverse piezoelectric effect. Under a constant $V_{\mathrm{GS}}$, larger $W_{\mathrm{O}}$ values result in smaller absolute values of $\sigma_{\mathrm{G}}$. Figure 6 also shows that both $\mu_{\mathrm{Total}}$ and $\mu_{\mathrm{PCF}}$ decrease as $V_{\mathrm{GS}}$ changes negatively, while $\mu_{\mathrm{DIS}}, \mu_{\mathrm{AP}}, \mu_{\mathrm{POP}}$ and $\mu_{\mathrm{IFR}}$ do not change with $V_{\mathrm{GS}}$. As greater negative $V_{\mathrm{GS}}$ amounts are applied, $\mu_{\mathrm{Total}}$ and $\mu_{\mathrm{PCF}}$ become closer in value. The relationship between electron mobility and $V_{\mathrm{GS}}$ can be explained as follows. For the narrowed open region of AlGaN/GaN HFETs, $n_{2 \mathrm{D}}$ does not change with $V_{\mathrm{GS}}$, which means that the scattering mechanisms other than PCF scattering do not change with $V_{\mathrm{GS}}$ either. As $V_{\mathrm{GS}}$ changes negatively, the absolute value of $\sigma_{\mathrm{G}}$ increases, the PCF scattering becomes stronger, and $\mu_{\mathrm{PCF}}$ decreases accordingly. When other scattering intensities remain unchanged, a decrease in $\mu_{\mathrm{PCF}}$ will inevitably lead to a decrease in $\mu_{\mathrm{Total}}$. On the other hand, when the negative $V_{\mathrm{GS}}$ value is large, the mobility of PCF scattering is obviously smaller than that of other scattering mechanisms (Fig. 6), which means that PCF scattering occupies a dominant position among the various types of scattering. Therefore, $\mu_{\text {Total }}$ and $\mu_{\mathrm{PCF}}$ are very similar in value. As $V_{\mathrm{GS}}$ continues to change negatively, the PCF scattering is further strengthened, and its proportion among all scattering mechanisms also increases. Therefore, greater negative $V_{\mathrm{GS}}$ values result in closer $\mu_{\text {Total }}$ and $\mu_{\mathrm{PCF}}$ values.

When comparing the $\mu_{\mathrm{PCF}}$ of samples 2,3 , and 4 , it can also be found that under the same $V_{\mathrm{GS}}$, smaller opening widths lead to smaller $\mu_{\mathrm{PCF}}$ in the open region. According to previous calculations, it is known that the absolute value of $\sigma_{\mathrm{G}}$ will increase as the opening width decreases. On the other hand, when the total width is the same, 

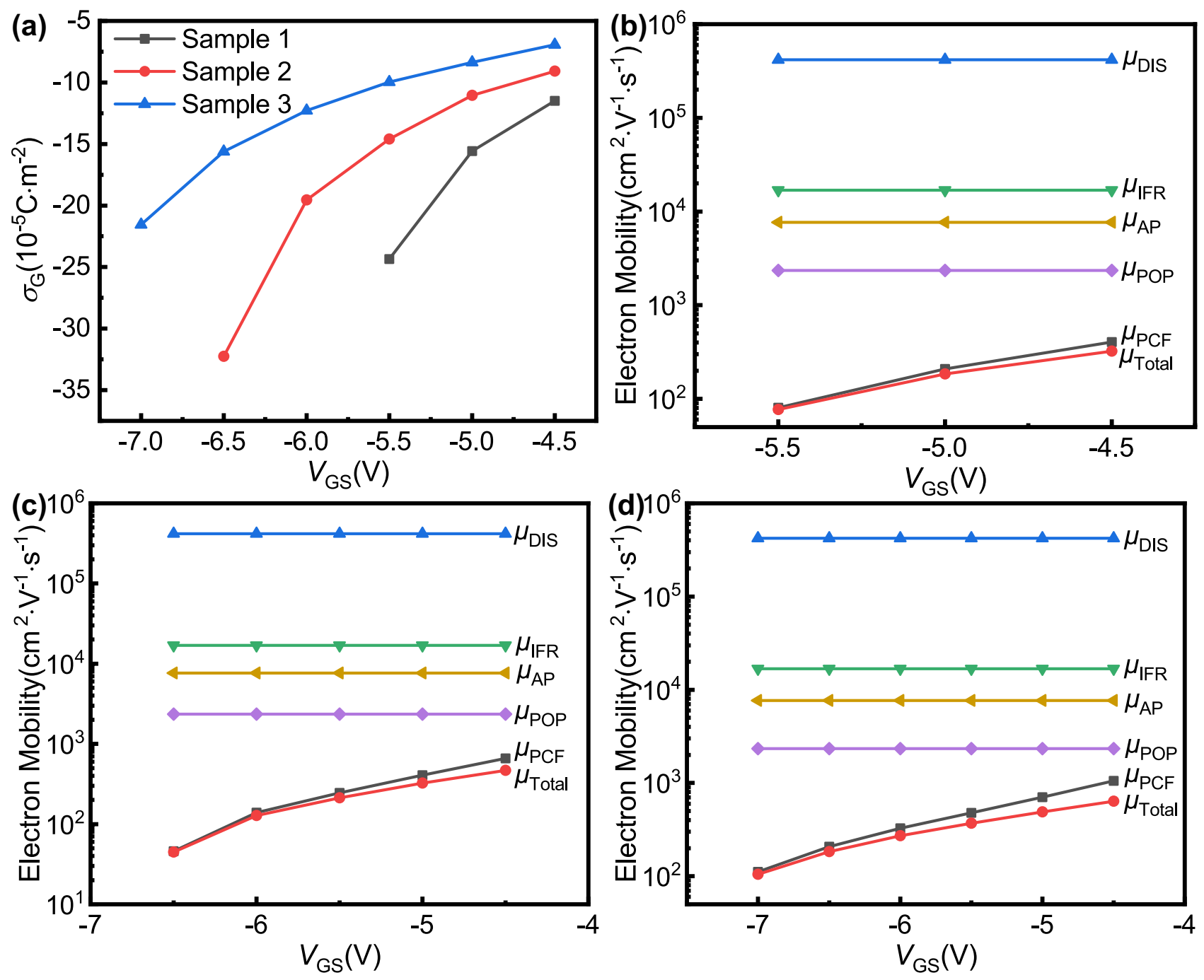

Figure 6. The calculated (a) additional polarization charge density for open-gate samples and various electron mobilities for (b) sample 2 , (c) sample 3 , and (d) sample 4 as a function of the gate bias. $\mu_{\mathrm{DIS}}, \mu_{\mathrm{IFR}}, \mu_{\mathrm{AB}} \mu_{\mathrm{POB}} \mu_{\mathrm{PCF}}$ and $\mu_{\text {Total }}$ correspond to the electron mobility of DIS scattering, IFR scattering, AP scattering, POP scattering, PCF scattering, and total electron mobility, respectively.

a smaller open region means a larger gate region. Therefore, as the opening width decreases, the total amount of additional polarization charges underneath the gate region will increase, and the total amount of electrons scattered in the open region will decrease. This will increase the PCF scattering, thereby reducing the $\mu_{\mathrm{PCF}}$ under the same $V_{\mathrm{GS}}$.

Through the above quantitative calculations, it can be further proven that for open-gate devices, PCF scattering plays an important role. When the 2DEG underneath the gate region is depleted, it is through PCF scattering, which changes the electron mobility in the open region, that $V_{\mathrm{GS}}$ can modulate $I_{\mathrm{DS}}$ and further realize the turn-off of the devices. Based on this mechanism, in order to improve the ability of $V_{\mathrm{GS}}$ to modulate $I_{\mathrm{DS}}$ in the open region, it is needed to find a way to enhance PCF scattering. Increasing the gate area to increase the additional polarization charge underneath the gate is a feasible method to enhance the PCF scattering, which is also the reason for choosing the large size device in this paper. The above research also shows that the modulation ability of $V_{\mathrm{GS}}$ to $I_{\mathrm{DS}}$ in the open region increases with the decrease of opening width. Therefore, if the gate length is reduced and the opening width is reduced in equal proportion, the same mechanism may be expected in devices with shorter gate length. Relevant research is currently in progress.

It has already been established that when $V_{\mathrm{DS}}$ is applied to AlGaN/GaN HFETs, electrons will be injected onto the surface of the device through the source and trapped by the trap states on the surface, resulting in a drop in the surface potential of the device ${ }^{39}$. In this way, there will be a negative potential difference between the surface and the channel, which is equivalent to a virtual gate with a negative bias being applied to the surface of the device to deplete the 2DEG in the channel. Therefore, the current saturation can be achieved through a mechanism similar to the channel pinch-off for ungated AlGaN/GaN HFETs, and it is also applicable to the open region of open-gate devices. In the above process, the virtual gate is formed by the influence of drain-source electric field, 

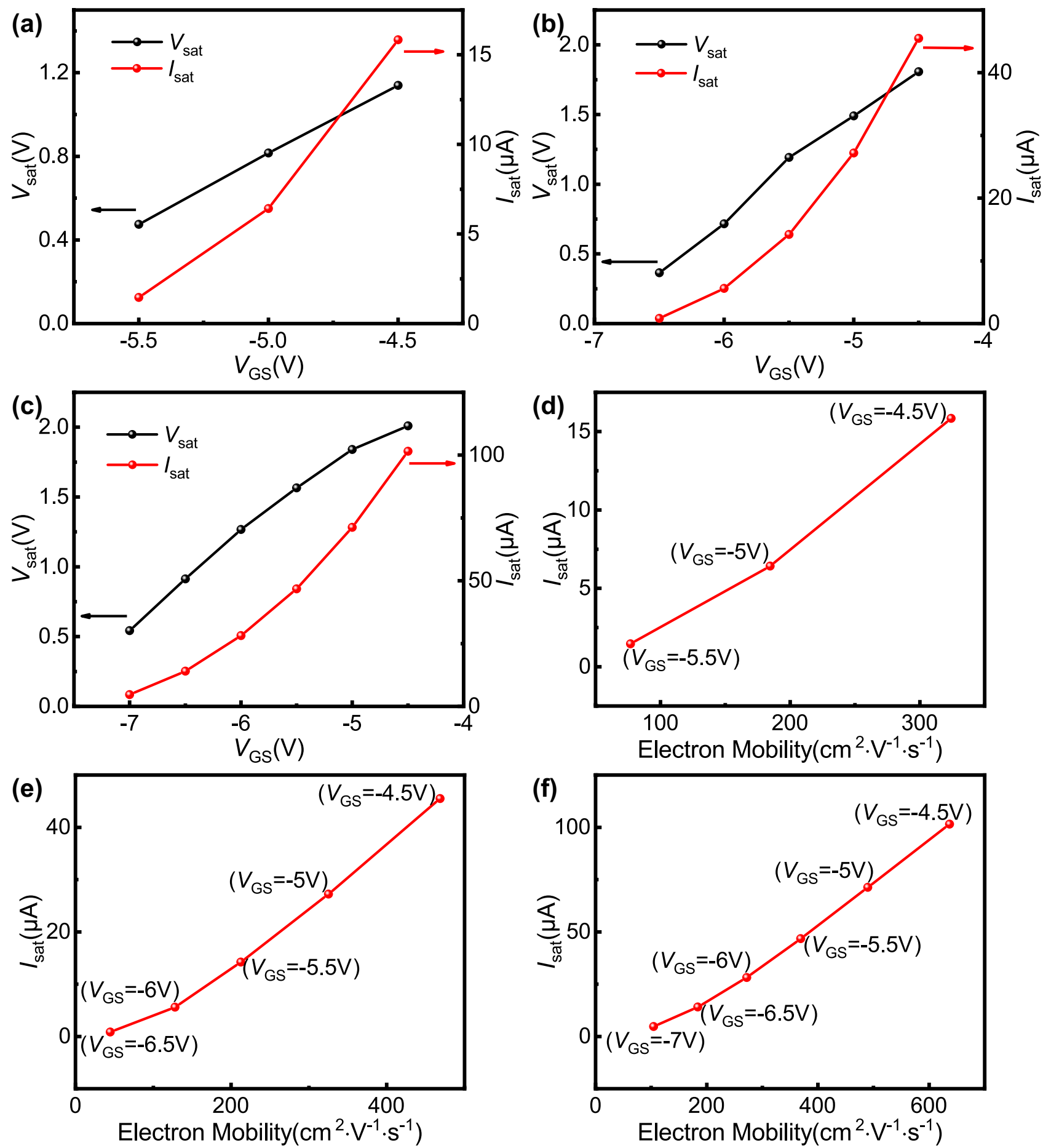

Figure 7. The measured saturation voltage and saturation current as a function of the gate bias for (a) sample 2, (b) sample 3, and (c) sample 4. The measured saturation current as a function of the calculated total electron mobility for (d) sample 2, (e) sample 3, and (f) sample 4. The saturation points are obtained by the method shown in Fig. 2. The electron mobility corresponds to the calculated total electron mobility shown in Fig. 6.

so its potential is not affected by gate bias theoretically. Therefore, this virtual gate can only affect the current saturation, but cannot be used to explain the modulation of gate bias on channel current.

Figure $7 \mathrm{a}-\mathrm{c}$ display the saturation voltage $\left(V_{\text {sat }}\right)$ and saturation current $\left(I_{\text {sat }}\right)$ values of samples 2,3 , and 4 as a function of $V_{\mathrm{GS}}$ when $V_{\mathrm{GS}} \leq-4.5 \mathrm{~V}$. $V_{\text {sat }}$ and $I_{\text {sat }}$ under different gate biases are obtained by the method shown in Fig. 2. It can be seen that the $V_{\text {sat }}$ and $I_{\text {sat }}$ values of each sample decrease as $V_{\mathrm{GS}}$ changes negatively. According to previous calculations, there is a decrease in electron mobility as $V_{\mathrm{GS}}$ changes negatively under the influence of PCF scattering, increasing the channel resistance. The conduction part of the device can be regarded as the series connection of the channel region resistance and the ohmic contact resistance, and the ohmic contact resistance does not change with $V_{\mathrm{Gs}}$. As $V_{\mathrm{GS}}$ changes negatively, the channel resistance increases, while the channel 
current decreases. This will lead to a reduction in the potential drop on the drain end ohmic contact resistance and further increase the channel potential. In this way, to reach the channel potential corresponding to the saturation point, the $V_{\mathrm{DS}}$ that needs to be provided will be reduced accordingly. Therefore, $V_{\text {sat }}$ will decrease as $V_{\mathrm{GS}}$ changes negatively. Additionally, the channel resistance will increase and $V_{\text {sat }}$ will decrease, inevitably leading to a decrease in $I_{\text {sat }}$. When $V_{\mathrm{GS}} \leq-4.5 \mathrm{~V}$, the relationships between $I_{\text {sat }}$ and electron mobility corresponding to different gate biases in the open region of samples 2, 3, and 4 are shown in Fig. $7 \mathrm{~d}-\mathrm{f}$. It can be seen that $I_{\text {sat }}$ and electron mobility are approximately linearly related, which is consistent with the paper of Kuzmík et al. ${ }^{39}$ As a result, the working mechanism of the open region can be further proven.

Application potential. The open-gate device has two conduction modes. When $V_{\mathrm{GS}} \leq-4.5 \mathrm{~V}$, only the open region of the device is conductive, and the conduction current is very low. At this time, $V_{\mathrm{GS}}$ influences the electron mobility through PCF scattering and further modulates $I_{\mathrm{DS}}$, which has a weaker modulation ability, but can still achieve effective modulation across a larger voltage range. Based on these characteristics of open-gate devices, it is believed that these devices may be suitable for a wide range of low power consumption class-A voltage amplifier applications. Taking sample 4 as an example, it is possible to make theoretical estimates of the performance parameters of the device as a class-A voltage amplifier.

A value of $V_{\mathrm{GS}}=-7.5$ to $-4.5 \mathrm{~V}$ was chosen as the working range of the amplifier, where only the open region of the device was turned on and $V_{\mathrm{GS}}$ had effective modulation of $I_{\mathrm{DS}}$, as seen from Fig. 2. The maximum current $\left(I_{\max }\right)$ of the device was the saturation current when $V_{\mathrm{GS}}=-4.5 \mathrm{~V}$, so $I_{\max }=1.02 \times 10^{-4} \mathrm{~A}$. The quiescent point was selected near the midpoint of the device's operating range so that the device was turned on for the entire period of the input sinusoidal signal. At this time, the amplitude of the input signal voltage $\left(v_{\text {in }}\right)$ was $1.5 \mathrm{~V}$. The amplitude of the maximum output voltage $\left(v_{\mathrm{om}}\right)$ was determined by the breakdown voltage $(B V)$ of the device ${ }^{40}$, which had been tested to be approximately $170 \mathrm{~V}$, so $v_{\mathrm{om}} \approx B V / 2=85 \mathrm{~V}$. Therefore, as a class-A voltage amplifier, the theoretical maximum value of the device voltage gain $\left(G_{\mathrm{m}}\right)$ can be obtained as $G_{\mathrm{m}}=v_{\mathrm{om}} / v_{\mathrm{in}} \approx 56.67$. In this case, the theoretical value of the direct current power $\left(P_{\mathrm{DC}}\right)$ of the device was determined by $I_{\max }$ and $B V$, expressed as ${ }^{40}$ $P_{\mathrm{DC}} \approx I_{\max } / 2 \times B V / 2=4.335 \mathrm{~mW} \approx 6.37 \mathrm{dbm}$. It can be seen that using an open-gate device as a class-A voltage amplifier can effectively amplify a larger input signal with very low power consumption.

As a separate voltage amplifier, the advantages of AlGaN/GaN open-gate HFETs mainly come from the lowtransconductance region with large voltage range and small current, which may be replaced by low-transconductance devices with other designs. However, voltage amplifiers usually need to be cascaded with other devices into circuit modules to realize their functions. At this time, the advantages of open-gate devices can be fully reflected. The open-gate device has no special requirements for the structure, doping and thickness of the material, and the preparation process is completely consistent with the normal device, so it can be prepared on the same heterojunction material as the normal device to facilitate circuit integration. If the normal gate structure is adopted, special material structure or process steps are usually required to achieve similar device characteristics, which is not conducive to the integration with normal devices. In addition, open-gate devices have two effective modulation regions: high-transconductance region and low-transconductance region, which is difficult to be realized by other device structures.

Due to their unique characteristics, open-gate devices may have many other applications, which we will continue to study in the future.

\section{Conclusions}

A novel AlGaN/GaN HFET based on open-gate technology was prepared, and a series of experimental tests and theoretical calculations were carried out. For this novel device, when the 2DEG underneath the gate region is depleted, $V_{\mathrm{GS}}$ can still effectively modulate $I_{\mathrm{DS}}$. By changing the width of the opening, the modulation of $V_{\text {th }}$ can easily be achieved. Related calculations have shown that the open-gate device has two working modes with different modulation abilities and working mechanisms. When $V_{\mathrm{GS}} \leq-4.5 \mathrm{~V}$, the working mechanism of the open-gate devices are related to PCF scattering and the virtual gate formed by surface electron injection. As a novel device with a new working mechanism, open-gate devices can be used as class-A voltage amplifiers with low power consumption, and also have broad prospects in other applications. Further research in the future will involve trying to reduce the size of the devices.

\section{Methods}

Sample fabrication. The $\mathrm{AlGaN} / \mathrm{GaN}$ heterostructure material used in the experiment was grown on a $350 \mu \mathrm{m} \mathrm{SiC} \mathrm{substrate.} \mathrm{Through} \mathrm{metal} \mathrm{organic} \mathrm{chemical} \mathrm{vapor} \mathrm{deposition} \mathrm{(MOCVD),} \mathrm{a} 2 \mu \mathrm{m}$ GaN buffer layer, $1.5 \mathrm{~nm}$ AlN insertion layer, $23 \mathrm{~nm} \mathrm{Al} \mathrm{n}_{0.25} \mathrm{Ga}_{0.75} \mathrm{~N}$ barrier layer, and $2 \mathrm{~nm} \mathrm{GaN}$ cap layer were grown in sequence. The sheet carrier density of the heterostructure material was about $7.41 \times 10^{12} \mathrm{~cm}^{-2}$. The device isolation was defined by inductively coupled plasma (ICP) etching. Using electron beam evaporation, $\mathrm{Ti} / \mathrm{Al} / \mathrm{Ni} / \mathrm{Au}$ was deposited successively as ohmic contacts, followed by rapid thermal annealing at $850{ }^{\circ} \mathrm{C}$ for $30 \mathrm{~s}$ in $\mathrm{N}_{2}$ atmosphere. The metal used for Schottky contact was $\mathrm{Ni} / \mathrm{Au}$, which was also deposited by electron beam evaporation. The patterns of device isolation, ohmic contact, and Schottky contact were all defined by UV-lithography.

Measurements. The output characteristics of the devices were measured at room temperature by a Keysight $\mathrm{B} 1500 \mathrm{~A}$ semiconductor parameter analyzer. The $\mathrm{C}-\mathrm{V}$ characteristics were also measured at room temperature using a Keysight B1520A at $1 \mathrm{MHz}$. 


\section{Data availability}

The data that support the findings of this study are available from the corresponding author upon reasonable request.

Received: 25 July 2021; Accepted: 8 November 2021

Published online: 17 November 2021

\section{References}

1. Mishra, U. K., Parikh, P. \& Wu, Y. F. AlGaN/GaN HEMTs-An overview of device operation and applications. Proc. IEEE. 90, 1022-1031 (2002).

2. Rajan, S., Xing, H. L., DenBaars, S., Mishra, U. K. \& Jena, D. AlGaN/GaN polarization-doped field-effect transistor for microwave power applications. Appl. Phys. Lett. 84, 1591-1593 (2004).

3. Saito, W. et al. High breakdown voltage undoped AlGaN-GaN power HEMT on sapphire substrate and its demonstration for DC-DC converter application. IEEE Trans. Electron Devices 51, 1913-1917 (2004).

4. Zhang, K. et al. High-linearity AlGaN/GaN FinFETs for microwave power applications. IEEE Electron Device Lett. 38, 615-618 (2017).

5. Gaska, R., Shur, M. S., Fjeldly, T. A. \& Bykhovski, A. D. Two-channel AlGaN/GaN heterostructure field effect transistor for high power applications. J. Appl. Phys. 85, 3009-3011 (1999).

6. Mi, M. et al. $90 \mathrm{~nm}$ gate length enhancement-mode AlGaN/GaN HEMTs with plasma oxidation technology for high-frequency application. Appl. Phys. Lett. 111, 173502 (2017).

7. Rashmi, Kranti, A., Haldar, S. \& Gupta, R. S. An accurate charge control model for spontaneous and piezoelectric polarization dependent two-dimensional electron gas sheet charge density of lattice-mismatched AlGaN/GaN HEMTs. Solid State Electron. 46, 621-630 (2002).

8. Cai, Y., Zhou, Y., Lau, K. M. \& Chen, K. J. Control of threshold voltage of AlGaN/GaN HEMTs by fluoride-based plasma treatment: From depletion mode to enhancement mode. IEEE Trans. Electron Devices 53, 2207-2215 (2006).

9. Pardeshi, H., Raj, G., Pati, S., Mohankumar, N. \& Sarkar, C. K. Influence of barrier thickness on AlInN/GaN underlap DG MOSFET device performance. Superlattices Microstruct. 60, 47-59 (2013).

10. Gregušová, D. et al. Adjustment of threshold voltage in AlN/AlGaN/GaN high-electron mobility transistors by plasma oxidation and $\mathrm{Al}_{2} \mathrm{O}_{3}$ atomic layer deposition overgrowth. Appl. Phys. Lett. 104, 013506 (2014).

11. Zhang, Y., Sun, M., Joglekar, S. J., Fujishima, T. \& Palacios, T. Threshold voltage control by gate oxide thickness in fluorinated GaN metal-oxide-semiconductor high-electron-mobility transistors. Appl. Phys. Lett. 103, 033524 (2013).

12. Sugiyama, T. et al. Threshold voltage control using $\mathrm{SiN}_{\mathrm{x}}$ in normally off AlGaN/GaN HFET with $\mathrm{p}-\mathrm{GaN}$ gate. Phys. Status Solidi C 7, 1980-1982 (2010).

13. Liu, Y. et al. The role of polarization coulomb field scattering in the electron mobility of AlGaN/AlN/GaN heterostructure fieldeffect transistors. J. Korean Phys. Soc. 68, 883-888 (2016).

14. Yang, M. et al. Effect of polarization coulomb field scattering on parasitic source access resistance and extrinsic transconductance in AlGaN/GaN heterostructure FETs. IEEE Trans. Electron Devices 63, 1471-1477 (2016).

15. Cui, P. et al. Influence of different gate biases and gate lengths on parasitic source access resistance in $\mathrm{AlGaN} / \mathrm{GaN}$ heterostructure FETs. IEEE Trans. Electron Devices 64, 1038-1044 (2017).

16. Cui, P., Lv, Y., Lin, Z., Fu, C. \& Liu, Y. Effect of polarization Coulomb field scattering on device linearity in AlGaN/GaN heterostructure field-effect transistors. J. Appl. Phys. 122, 124508 (2017).

17. Luan, C. et al. Influence of polarization coulomb field scattering on the subthreshold swing in depletion-mode AlGaN/AlN/GaN heterostructure field-effect transistors. Phys. E 62, 76-79 (2014).

18. Binari, S. C. et al. Trapping effects and microwave power performance in AlGaN/GaN HEMTs. IEEE Trans. Electron Devices 48 , 465-471 (2001).

19. Fu, L. et al. Field-dependent carrier trapping induced kink effect in AlGaN/GaN high electron mobility transistors. Appl. Phys. Lett. 98, 173508 (2011)

20. Wang, M. \& Chen, K. J. Kink effect in AlGaN/GaN HEMTs induced by drain and gate pumping. IEEE Electron Device Lett. 32, 482-484 (2011).

21. Wieck, A. D. \& Ploog, K. In-plane-gated quantum wire transistor fabricated with directly written focused ion beams. Appl. Phys. Lett. 56, 928-930 (1990).

22. Santoruvo, G. \& Matioli, E. In-plane-gate GaN transistors for high-power RF applications. IEEE Electron Device Lett. 38, 1413-1416 (2017).

23. Thornton, T. J., Pepper, M., Ahmed, H., Andrews, D. \& Davies, G. J. One-dimensional conduction in the 2D electron gas of a GaAs-AlGaAs heterojunction. Phys. Rev. Lett. 56, 1198-1201 (1986).

24. van Wees, B. J. et al. Quantized conductance of point contacts in a two-dimensional electron gas. Phys. Rev. Lett. 60, 848-850 (1988).

25. Zheng, H. Z., Wei, H. P., Tsui, D. C. \& Weimann, G. Gate-controlled transport in narrow GaAs/ $\mathrm{Al}_{\mathrm{x}} \mathrm{Ga}_{1-\mathrm{x}} \mathrm{As}$ heterostructures. Phys. Rev. B 34, 5635-5638 (1986).

26. Laux, S. E., Frank, D. J. \& Stern, F. Quasi-one-dimensional electron states in a split-gate GaAs/AlGaAs heterostructure. Surf. Sci. 196, 101-106 (1988)

27. Vetury, R., Zhang, N., Keller, S. \& Mishra, U. K. The impact of surface states on the DC and RF characteristics of AlGaN/GaN HFETs. IEEE Trans. Electron Devices 48, 560-566 (2001).

28. Hsu, L. \& Walukiewicz, W. Electron mobility in $\mathrm{Al}_{\mathrm{x}} \mathrm{Ga}_{1-\mathrm{x}} \mathrm{N} / \mathrm{GaN}$ heterostructures. Phys. Rev. B 56, 1520-1528 (1997).

29. Antoszewski, J. et al. Scattering mechanisms limiting two-dimensional electron gas mobility in $\mathrm{Al}_{025} \mathrm{Ga}_{0.75} \mathrm{~N} / \mathrm{GaN}$ modulationdoped field-effect transistors. J. Appl. Phys. 87, 3900-3904 (2000).

30. Lv, Y. et al. Polarization Coulomb field scattering in AlGaN/AlN/GaN heterostructure field-effect transistors. Appl. Phys. Lett. 98, $123512(2011)$

31. Bernardini, F., Fiorentini, V. \& Vanderbilt, D. Spontaneous polarization and piezoelectric constants of III-V nitrides. Phys. Rev. B 56, 10024-10027 (1997).

32. Romanov, A. E., Baker, T. J., Nakamura, S. \& Speck, J. S. Strain-induced polarization in wurtzite III-nitride semipolar layers. J. Appl. Phys. 100, 023522 (2006).

33. Anwar, A., Webster, R. T. \& Smith, K. V. Bias induced strain in AlGaN/GaN heterojunction field effect transistors and its implications. Appl. Phys. Lett. 88, 203510 (2006).

34. Zhao, J. et al. Electron mobility related to scattering caused by the strain variation of AlGaN barrier layer in strained AlGaN/GaN heterostructures. Appl. Phys. Lett. 91, 173507 (2007).

35. Luan, C. et al. Theoretical model of the polarization Coulomb field scattering in strained AlGaN/AlN/GaN heterostructure fieldeffect transistors. J. Appl. Phys. 116, 044507 (2014). 
36. Gurusinghe, M. N., Davidsson, S. K. \& Andersson, T. G. Two-dimensional electron mobility limitation mechanisms in $\mathrm{Al}_{\mathrm{x}} \mathrm{Ga}_{1-\mathrm{x}} \mathrm{N} /$ GaN heterostructures. Phys. Rev. B 72, 045316 (2005).

37. Cui, P. et al. Effect of different gate lengths on polarization coulomb field scattering potential in AlGaN/GaN heterostructure field-effect transistors. Sci. Rep. 8, 9036 (2018).

38. Cui, P., Lin, Z., Fu, C., Liu, Y. \& Lv, Y. A method to determine electron mobility of the two-dimensional electron gas in AlGaN/ GaN heterostructure field-effect transistors. Superlattices Microstruct. 110, 289-295 (2017).

39. Kuzmik, J., Bychikhin, S., Pogany, D., Gaquiere, C. \& Morvan, E. Current conduction and saturation mechanism in AlGaN/GaN ungated structures. J. Appl. Phys. 99, 123720 (2006).

40. Bahl, I. J. Fundamentals of RF and Microwave Transistor Amplifiers Ch 8 (Wiley, 2008).

\section{Acknowledgements}

This work was supported by the National Natural Science Foundation of China (Grant Nos. 11974210 and 11574182).

\section{Author contributions}

Y.L. and Z.L. contributed to the research design, experiment measurements, data analysis, and manuscript preparation. Y.L. fabricated the device. S.G. designed the layout. Z.L. and A.C. carried out the mathematical calculation. Y.Y., G.J., and Y.Z. provided scientific advice. All authors reviewed this manuscript.

\section{Competing interests}

The authors declare no competing interests.

\section{Additional information}

Supplementary Information The online version contains supplementary material available at https:/doi.org/ 10.1038/s41598-021-01917-9.

Correspondence and requests for materials should be addressed to Z.L.

Reprints and permissions information is available at www.nature.com/reprints.

Publisher's note Springer Nature remains neutral with regard to jurisdictional claims in published maps and institutional affiliations.

(c) Open Access This article is licensed under a Creative Commons Attribution 4.0 International License, which permits use, sharing, adaptation, distribution and reproduction in any medium or format, as long as you give appropriate credit to the original author(s) and the source, provide a link to the Creative Commons licence, and indicate if changes were made. The images or other third party material in this article are included in the article's Creative Commons licence, unless indicated otherwise in a credit line to the material. If material is not included in the article's Creative Commons licence and your intended use is not permitted by statutory regulation or exceeds the permitted use, you will need to obtain permission directly from the copyright holder. To view a copy of this licence, visit http://creativecommons.org/licenses/by/4.0/.

(C) The Author(s) 2021 